\title{
Modelagem Computacional do Acoplamento Hidro-Geomecânico em Prospecção Secundária de Petróleo Aplicado ao Pré-Sal Brasileiro
}

\author{
Luiz C. Radtke ${ }^{1}$ \\ Departamento de Matemática, UFRN, Natal, RN \\ Marcio A. Murad ${ }^{2}$ \\ Laboratório Nacional de Computação Científica, LNCC, Petrópolis, RJ
}

\begin{abstract}
Resumo. Neste trabalho propomos o desenvolvimento de um novo modelo computacional para descrever o acoplamento hidro-geomecânico durante o processo de recuperação de petróleo por injeção de água nas formações geológicas que compõem o pré-sal brasileiro. A descrição do acoplamento entre hidrodinâmica, transporte bifásico não linear e geomecânica de rochas salinas viscoelásticas é construída no contexto de formulações iterativamente acopladas, onde o problema é decomposto em vários subsistemas associados a cada fenômeno físico. Os subsistemas governantes do movimento dos fluidos são discretizados por métodos numéricos localmente conservativos e o subsistema da geomecânica não linear pelo método dos elementos finitos mistos em conjunção com algoritmos de predição-correção. Simulações numéricas em geometrias fornecidas pela sísmica do pré-sal são realizadas ilustrando precisamente a influência dos efeitos geomecânicos sobre as curvas de produção.
\end{abstract}

Palavras-chave. Modelagem Matemática, Acoplamento Hidro-Geomecânico, Métodos Numéricos

\section{Introdução}

A recente descoberta de petróleo localizado abaixo de uma espessa camada de sal, conhecida como formação geológica do pré-sal brasileiro, representa um marco histórico para a exploração de petróleo no Brasil. Esta reserva é a maior já descoberta abrangendo cerca de 800 quilômetros ao longo da costa sudeste do país, onde o óleo reside em rochas carbonáticas localizadas a 7.000 metros abaixo do nível do mar subjacente à formação salina (evaporito). Por outro lado, a exploração de petróleo no pré-sal apresenta vários desafios. Além da exploração em águas muito profundas, a camada de sal acima do reservatório oferece baixa resolução sísmica, dificultando a obtenção de imagens nítidas. Tal fato, aliado ao alto teor de heterogeneidade dos carbonatos, conduz à uma alta complexidade na caracterização e avaliação das formações.

\footnotetext{
${ }^{1}$ luizcr@ccet.ufrn.br

2 murad@lncc.br
} 
Com a descoberta do pré-sal em águas profundas, o estudo detalhado para descrever o acoplamento entre mecânica e hidrodinâmica de escoamentos multifásicos em meios porosos deformáveis torna-se fundamental na análise do processo de recuperação secundária de hidrocarbonetos [1]. A modelagem do acoplamento hidro-geomecânico é usualmente apresentada na literatura sob duas formas conhecidas como: formulação totalmente acoplada e sequencial. Na formulação totalmente acoplada o sistema de equações proveniente do modelo hidro-geomecânico é resolvido simultaneamente impondo-se a mesma escala de tempo para os dois subsistemas (hidrodinâmico e geomecânico), envolvendo o armazenamento de matrizes maiores e demandando maior esforço computacional na resolução do sistema linear resultante [3]. Além disso, a formulação completamente acoplada não é naturalmente estendida para descrever as rochas adjacentes impermeáveis, uma vez que nestes sub-domínios necessita-se resolver somente a geomecânica. Nos métodos sequenciais o sistema de equações é dividido em subsistemas que são resolvidos de maneira escalonada em relação ao tempo, onde o acoplamento é realizado da forma sequencial através dos termos de fonte tratados iterativamente entre os subsistemas. Neste contexto, destacam-se as formulações iterativamente acopladas cuja estabilidade e convergência dos algoritmos foram discutidas em [6].

No desenvolvimento deste trabalho, procuramos representar de forma mais realista possível as formações geológicas do pré-sal e as rochas capeadoras incorporadas no modelo via camadas de sal e pós-sal. A camada de sal é tratada como um meio viscoelástico não-linear governado por uma lei de potência de Norton para a deformação viscosa [7]. A camada do pós-sal impermeável e o reservatório são considerados elásticos lineares.

\section{Modelagem Matemática do Acoplamento Hidro-Geome- cânico}

Para a dedução do modelo matemático o domínio do problema foi subdividido em três regiões, conforme mostrado na Figura 1.

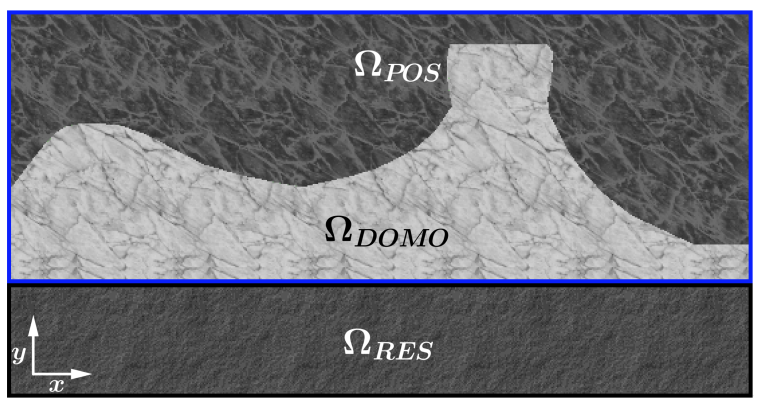

Figura 1: Partição do domínio $\Omega$ em três subdomínios $\Omega_{P O S}, \Omega_{D O M O}$ e $\Omega_{R E S}$.

Sejam $\Omega_{R E S}, \Omega_{D O M O}$ e $\Omega_{P O S} \in \mathbb{R}^{2}$ os domínios ocupados pelo carbonato, domo salino viscoelástico e o pós-sal elástico linear. Conhecidas as constantes $\mathcal{C}, \rho_{w}, \rho_{o}$, $\rho_{s}^{P O S}, \rho_{s}^{D O M O}, \rho_{s}^{R E S}, \mathcal{E}_{R}^{*}, \sigma_{R}, N$, os campos de tensão e pressão iniciais $\boldsymbol{\sigma}^{0}, p^{0}$ e as 
funções $\lambda_{w}\left(S_{w}\right), \lambda_{o}\left(S_{w}\right), \lambda_{t}\left(S_{w}\right), K(\phi), \boldsymbol{\eta}(\phi)$, encontrar os campos de pressão $p(\mathbf{x}, t)$, tensão efetiva $\boldsymbol{\sigma}_{e f}(\mathbf{x}, t)$, deslocamentos $\mathbf{u}(\mathbf{x}, t)$, saturação da água $S_{w}(\mathbf{x}, t)$, porosidade $\phi(\mathbf{x}, t)$ e velocidade de Darcy $\mathbf{v}_{D t}(\mathbf{x}, t)$ com $\mathbf{x} \in \Omega, t \in[0, T]$ satisfazendo

$$
\begin{aligned}
& \left\{\begin{array}{l}
\operatorname{div} \boldsymbol{\sigma}_{e f}+\rho_{s}^{P O S} \boldsymbol{g}=0 \\
\boldsymbol{\sigma}_{e f}=\mathcal{C} \mathcal{E}_{e}+\boldsymbol{\sigma}^{0} \\
\mathcal{E}_{e}=\frac{1}{2}\left(\nabla \mathbf{u}+(\nabla \mathbf{u})^{T}\right)
\end{array}\right. \\
& \left\{\begin{array}{l}
\operatorname{div} \boldsymbol{\sigma}_{e f}+\rho_{s}^{D O M O} \boldsymbol{g}=0 \\
\boldsymbol{\sigma}_{e f}=\mathcal{C} \mathcal{E}_{e}+\boldsymbol{\sigma}^{0} \\
\mathcal{E}_{e}=\frac{1}{2}\left(\nabla \mathbf{u}+(\nabla \mathbf{u})^{T}\right) \\
\mathcal{E}=\mathcal{E}_{e}+\mathcal{E}_{v} \\
\sigma_{V}=\sqrt{\frac{3}{2} \mathbf{S}: \mathbf{S}} \\
\mathbf{S}=2 G \boldsymbol{P}_{d e v} \mathcal{E}_{e} \\
\dot{\mathcal{E}}_{v}=\dot{\gamma} \frac{\partial \sigma_{V}}{\partial \sigma}=\dot{\gamma} \sqrt{\frac{3}{2}} \frac{\mathbf{S}}{\|\mathbf{S}\|} \\
\dot{\gamma}=\mathcal{E}_{R}^{*}\left(\frac{\sigma_{V}}{\sigma_{R}}\right)^{N}
\end{array}\right.
\end{aligned}
$$

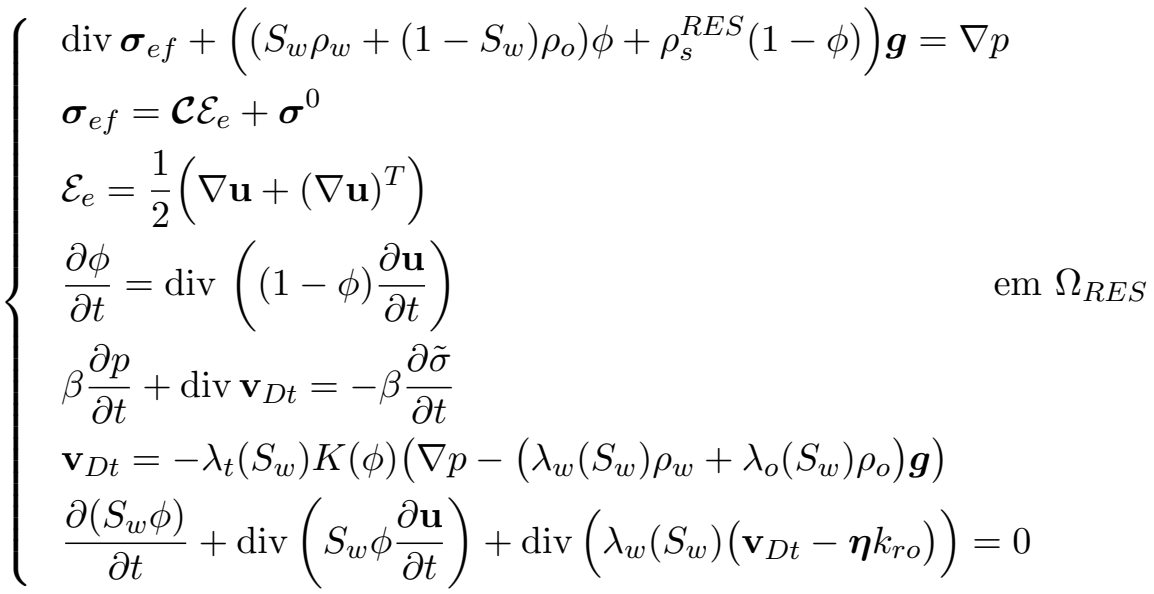

com condições iniciais

$$
\begin{array}{cc}
\operatorname{div}(\mathbf{u}(\mathbf{x}, 0))=0, & \phi(\mathbf{x}, 0)=\phi^{0}(\mathbf{x}), \\
\boldsymbol{\sigma}_{e f}(\mathbf{x}, 0)=\boldsymbol{\sigma}^{0}(\mathbf{x}), & S_{w}(\mathbf{x}, 0)=S_{w}^{0}(\mathbf{x}),
\end{array}
$$

e condições de contorno

$$
\begin{array}{cc}
\mathbf{u}(\mathbf{x}, t)=0 \text { sobre } \Gamma_{D}^{u}, & \boldsymbol{\sigma}_{T} \boldsymbol{n}=H(\mathbf{x}) \text { sobre } \Gamma_{N}^{u}, \\
p(\mathbf{x}, t)=0 \text { sobre } \Gamma_{D}^{p}, & \mathbf{v}_{D t} \cdot \boldsymbol{n}=Q(\mathbf{x}) \text { sobre } \Gamma_{N}^{p}, \\
\mathbf{v}_{D t} \cdot \boldsymbol{n}=0 \text { sobre } \Gamma-\left(\Gamma_{N}^{p} \cup \Gamma_{D}^{p}\right), & S_{w}(\mathbf{x}, t)=s(\mathbf{x}) \text { sobre } \Gamma_{N}^{p},
\end{array}
$$


onde

$$
\begin{array}{ll}
\Gamma_{D}^{u} \cap \Gamma_{N}^{u}=\emptyset, & \Gamma_{D}^{u} \cup \Gamma_{N}^{u}=\Gamma, \\
\Gamma_{D}^{p} \cap \Gamma_{N}^{p}=\emptyset, & \Gamma_{D}^{p} \cup \Gamma_{N}^{p}=\Gamma .
\end{array}
$$

Na resolução numérica dos subsistemas de EDP's, adotamos a formulação iterativamente acoplada, utilizando uma malha estendida para o módulo geomecânico, incluindo o particionamento do domínio ocupado pelas rochas capeadoras. A formulação iterativamente acoplada dá origem a três subsistemas que serão discretizados através de métodos numéricos adequados. Para a evolução temporal do modelo, utilizamos um esquema baseado em IMPES (implícito na pressão e geomecânica e explicito na saturação com passo de tempo limitado pela condição CFL). Para a equação hiperbólica da saturação, propomos o método de volumes finitos de mais alta ordem baseado em esquemas centrais KT desenvolvido por [8]. Para o subsistema hidrodinâmico utilizamos os métodos de elementos finitos mistos fazendo uso dos elementos de Raviart-Thomas de mais baixa ordem [9]. Finalmente para discretização do problema geomecânico, o qual é resolvido na malha estendida incluindo as rochas capeadoras, propomos a utilização de formulações mistas, mais precisamente o método Bbar proposto por [10].

\section{Simulações Numéricas}

Nesta seção para validarmos a formulação iterativamente acoplada iniciamos apresentando o problema 1D de Terzaghi, o qual consiste de um meio poroso saturado por um único fluido que ocupa o domínio $\Omega$ com fronteiras $\Gamma_{d}, \Gamma_{i}, \Gamma_{s}$ impermeáveis e uma condição de Dirichlet homogênea prescrita para o deslocamento na direção $y$ nas fronteiras $\Gamma_{i}$ e $\Gamma_{s}$ e em ambas as direções $x$ e $y$ na fronteira $\Gamma_{d}$. Na fronteira $\Gamma_{e}$, o reservatório é submetido a uma condição de carregamento de Neumann e, simultaneamente, uma condição de Dirichlet homogênea é imposta para a poro-pressão. O problema na forma variacional pode ser visto como um caso particular da formulação hidro-geomecânica proposta e nestas condições o problema possuí solução analítica [11].
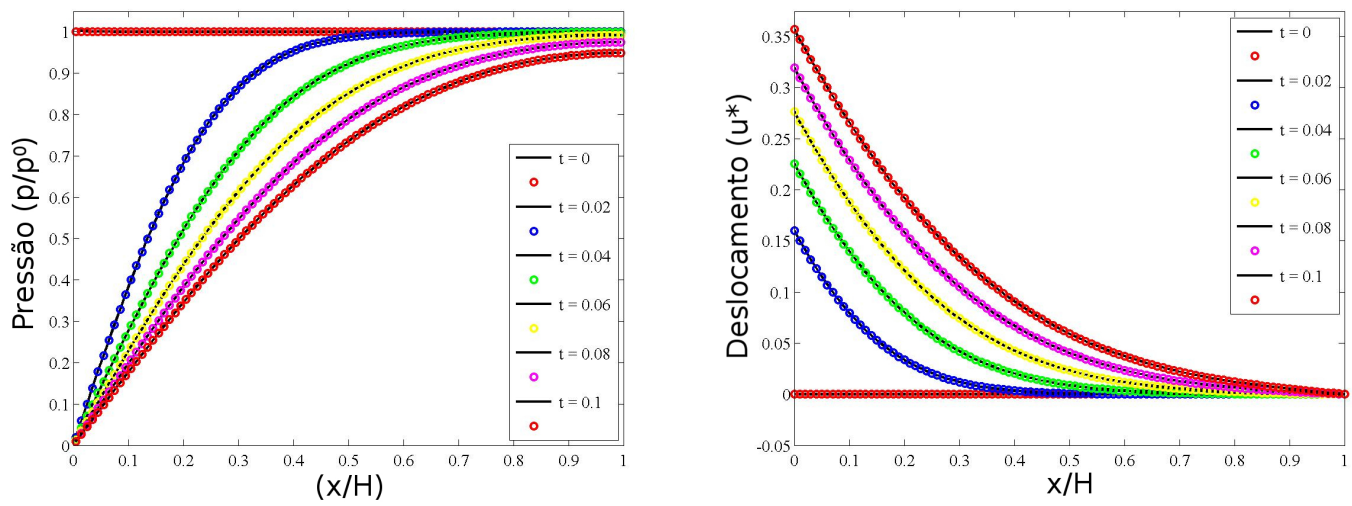

Figura 2: Comparação entre as soluções analítica (linha contínua) e a numérica (linha pontilhada) para deslocamento e pressão do problema de Terzaghi. 
Comparando as soluções, analíticas e numéricas, observamos proximidade entre elas na pressão e no deslocamento, demostrando a eficiência do método numérico adotado.

Procedemos agora rumo às simulações numéricas do processo de recuperação secundária de petróleo através da injeção de água no carbonato residente no pré-sal brasileiro. Na Figura 3 são apresentadas as curvas de produção de óleo, comparando os casos de matriz deformável e rígida. Podemos observar que para meio poroso rígido a produção de óleo mantém-se contante e igual à quantidade de água injetada $\left(100 \mathrm{~m}^{3} /\right.$ dia $)$, até a chegada da água no poço de produção, quando a produção de óleo decai abruptamente. Ao considerarmos o meio deformável, o movimento da fase sólida faz com que o volume do óleo produzido não corresponda à mesma quantidade de água injetada. Neste cenário, podemos observar a formação de quatro regimes distintos. Inicialmente, ocorre um pico de produção devido à abertura do poço produtor, após o início do processo de injeção de água, identificamos o segundo regime, onde a produção de óleo é inferior à quantidade de água injetada. Por volta de 6000 dias, tem início o terceiro regime, onde a produção de óleo supera a quantidade de água injetada, devido ao efeito de compactação da matriz porosa. Quando a água chega ao poço de produção Neste momento, inicia-se o quarto regime, caracterizado pelo decrescimento abrupto da produção de óleo.

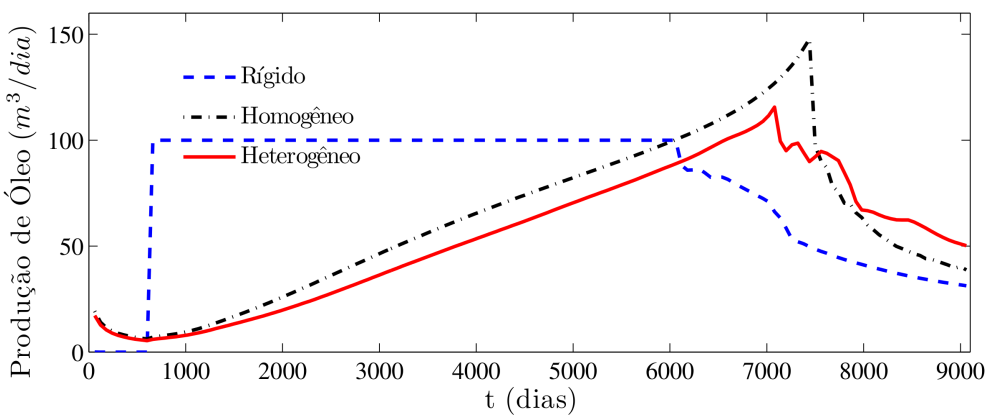

Figura 3: Comparação entre as curvas de produção para os casos rígido e deformável (homogêneo e heterogêneo).

Ao compararmos as curvas de produção de óleo para os casos homogêneo e heterogêneo, constatamos que a frente de água chega antecipadamente no poço de produção no caso heterogêneo, efeito advindo da formação de fingers oriundos da heterogeneidade aliada à razão de viscosidade entre a água e o óleo. A formação dos fingers acarreta uma produção de óleo, no caso heterogêneo, ligeiramente inferior do que no caso homogêneo.

Dando continuidade às simulações, na Figura 4 são apresentados os perfis de saturação do fluido injetado e as tensões de Von Mises na formação salina para o caso típico de prospecção secundária, onde o reservatório encontra-se inicialmente saturado por óleo e é injetado água para forçar a percolação do óleo. Ao iniciar o processo de injeção, observamos que o efeito da heterogeneidade causa um espalhamento da frente de onda, este efeito é fortemente influenciado pela razão de viscosidade, a qual tende a aumentar a instabilidade da interface entre os dois fluidos. Observamos também que a razão entre as viscosidades influência consideravelmente nas tensões de Von Mises geradas pelo processo de injeção. 
Dado que o óleo apresenta uma maior viscosidade quando comparado à água, a permeabilidade relativa do fluido injetado é menor, gerando assim maior resistência a percolação, e com isso aumentando a pressão no poço de injeção e, consequentemente, gerando tensões mais elevadas na matriz porosa e na rocha capeadora adjacente ao reservatório.

Ao evoluirmos no tempo, podemos observar que à medida que nos afastamos do poço de injeção, o efeito do empuxo torna-se mais expressivo, fazendo com que o fluido injetado escoe pela parte superior do reservatório.
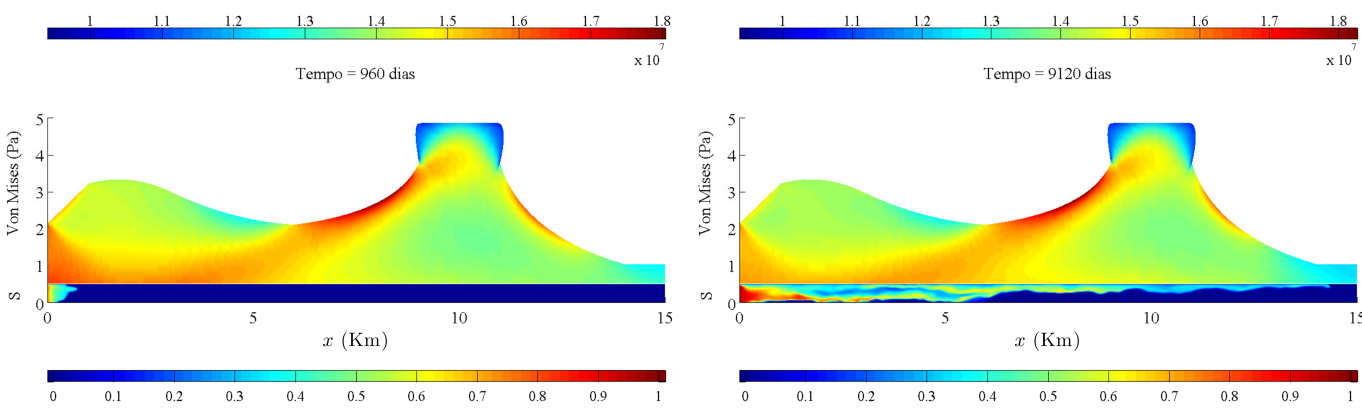

Figura 4: Evolução da saturação e da tensão de Von Mises.

\section{Conclusões}

Neste trabalho, construímos um novo modelo computacional para descrever o acoplamento hidro-geomecânico em processos de recuperação secundária nas formações geológicas que compõem o pré-sal brasileiro. Ao contrário dos modelos hidro-geomecânicos clássicos, baseados na formulação completamente acoplada, a abordagem proposta foi desenvolvida no contexto da formulação iterativamente acoplada, que exibe vantagem de explorar a disparidade entre as escalas de tempo dos fenômenos envolvidos, obtendo redução significativa do esforço computacional, além da flexibilidade de projetar métodos numéricos adequados na discretização de cada subsistema.

Simulações numéricas mostraram regimes distintos de produção caracterizados pelo decréscimo da produção inicial, devido à expansão da rocha, seguido de um acréscimo significativo, induzido pelo mecanismo de compaction drive e de uma queda abrupta no tempo de chegada da água no poço de produção.

Em suma, a formulação iterativamente acoplada proposta neste trabalho mostrouse bastante promissora para descrever processos de recuperação secundária no pré-sal brasileiro.

\section{Referências}

[1] M. A. Murad, A. J. Obregon, T. V. Lopes, E. Garcia, L. C. Radtke, M. R. Correa, M. R. Borges, and S. A. de Lima, "New locally conservative numerical schemes for 
hydrogeomechanical couplings in strongly heterogeneous presalt reservoirs," Society of Petroleum Engineers, 2013.

[2] M. D. Zobach, Reservoir Geomechanics. Cambridge Press, 2010.

[3] M. A. Murad, M. Borges, J. A. Obregon, and M. Correa, "A new locally conservative numerical method for two-phase flow in heterogeneous poroelastic media," Computers and Geotechnics, vol. 48, no. 0, pp. 192 - 207, 2013.

[4] A. Settari and D. Walters, "Advances in coupled geomechanical and reservoir modeling with applications to reservoir compaction," SPE Reservoir Simulation Symposium, pp. 14-17, Jan. 1999.

[5] D. Tran, A. Settari, and L. Nghiem, "New iterative coupling between a reservoir simulator and a geomechanics module," SPE Journal, vol. 9-3, pp. 362-369, 2004.

[6] J. Kim, H. Tchelepi, and R. Juanes, "Stability and convergence of sequential methods for coupled flow and geomechanics: drained and undrained splits," Comput. Methods Appl. Mech. Engrg., vol. 200, no. 23-24, pp. 2094-2116, 2011.

[7] A. M. Costa and E. P. Junior, Sal: Geologia e Tectônica, ch. Rocha Salina na Indústria do Petróleo: aspectos relacionados á reologia e á perfuração de rochas salinas, pp. 362 - 385. São Paulo: Beca, $2^{a}$ ed., 2008.

[8] A. Kurganov and E. Tadmor, "New high-resolution central schemes for nonlinear conservation laws and convection-diffusion equations," Journal of Computational Physics, vol. 160, no. 1, pp. $241-282,2000$.

[9] P. A. Raviart and J. M. Thomas, "A mixed finite element method for second order elliptic problems," vol. 606, pp. 292-315, 1977. 10.1007/BFb0064470.

[10] T. Hughes, The Finite Element Method: Linear Static and Dynamic Finite Element Analysis. Dover Civil and Mechanical Engineering, Dover Publications, 2000.

[11] M. A. Murad and A. F. D. Loula, "On stability and convergence of finite element approximations of biot's consolidation problem," International Journal for Numerical Methods in Engineering, vol. 37, no. 4, pp. 645-667, 1994. 